\section{Service industry}

\section{Annual Business Inquiry}

(Tables 24.1, 24.3 and 24.4)

For details of the Annual Business Inquiry, see the text accompanying Table 22.1.

\section{Retail trade: index numbers of value and volume}

\section{(Table 24.2)}

The main purpose of the Retail Sales Inquiry (RSI) is to provide up-to-date information on short period movements in the level of retail sales. In principle, the RSI covers the retail activity of every business classified in the retail sector (Division 52 of the 2003 Standard Industrial Classification) in Great Britain. A business will be classified to the retail sector if its main activity is one of the individual 4 digit SIC categories within Division 52. The retail activity of a business is then defined by its retail turnover, that is, the sale of all retail goods (note that petrol, for example, is not a retail good).

The Retail Sales Index is compiled from the information returned to the statutory inquiries into the distribution and services sector. The inquiry is addressed to a stratified sample of 5,000 businesses classified to the retail sector, the stratification being by 'type of store' (the individual 4 digit SIC categories within Division 52) and by size. The sample structure is designed to ensure that the inquiry estimates are as accurate as possible. In terms of the selection, this means that:

- each of the individual 4 digit SIC categories are represented, their coverage depending upon the relative size of the category and the variability of the data

- within each 4 digit SIC category, the larger retailers tend to be fully enumerated with decreasing proportions of medium and smaller retailers

The structure of the inquiry is updated periodically, by reference to the more comprehensive results of the Annual Business Inquiry ( $\mathrm{ABI}$ ). The monthly inquiry also incorporates a rotation element for the smallest retailers. This helps to spread the burden more fairly, as well as improving the representativeness between successive benchmarks.

During 2003, the Retail Sales Index was rebased using detailed information from the 2000 Annual Business Inquiry. The reference year is currently set at $2000=100$. A review of the retail Sales Inquiry was published in October 2008. The findings are available at: www.statistics.gov.uk/StatBase/ Product.asp?vlnk=13527

The latest summary statistics are published each month by First Release. More disaggregated indices (not seasonally adjusted) are published each month in the Business Monitor SDM28. See: www.statistics.gov.uk/rsi 
Service industry

\section{Retail businesses $^{1}$ \\ United Kingdom}

$£$ million and percentages

\begin{tabular}{|c|c|c|c|c|c|c|}
\hline & & 2002 & 2003 & 2004 & 2005 & 2006 \\
\hline Number of businesses & $\mathrm{ZABE}$ & 207513 & 202604 & 200606 & 201419 & 200004 \\
\hline Total turnover $^{2}$ & ZABL & 265577 & 278373 & 288716 & 295952 & 310150 \\
\hline Value Added Tax in total turnover & ZABM & 26907 & 28505 & 29420 & 29923 & 31255 \\
\hline Retail turnover $^{2}$ & ZABN & 238456 & 250849 & 258903 & 264427 & 277298 \\
\hline Non-retail turnover ${ }^{2}$ & ZABO & 27121 & 27524 & 29812 & 31525 & 32852 \\
\hline Value of commercial insurance claims received & $\mathrm{ZABP}$ & 105 & 65 & 40 & 76 & 81 \\
\hline Subsidies received from UK government sources & & & & & & \\
\hline and the EC & ZAEN & 4 & 5 & 10 & 16 & 10 \\
\hline Employment costs ${ }^{3}$ & $\mathrm{ZABQ}$ & 29779 & 31367 & 32806 & 34729 & 36035 \\
\hline Gross wages and salaries & ZABR & 26933 & 28294 & 29481 & 30938 & 32197 \\
\hline Redundancy and severance payments & ZABS & 130 & 134 & 158 & 251 & 164 \\
\hline Employers' National Insurance contributions & ZABT & 1805 & 1991 & 2142 & 2282 & 2393 \\
\hline Contributions to pension funds & $\mathrm{ZABU}$ & 911 & 948 & 1026 & 1258 & 1280 \\
\hline Increase during year & ZABV & 1284 & 978 & 957 & 726 & 887 \\
\hline Value at end of year & ZABW & 22400 & 23024 & 23527 & 24211 & 25120 \\
\hline Total turnover ${ }^{3}$ divided by end-year stocks (Quotient) & $\mathrm{ZABX}$ & 10.7 & 10.9 & 11.0 & 11.0 & 11.1 \\
\hline Purchases of goods, materials and services ${ }^{3}$ & ZABY & 185875 & 194169 & 199773 & 205399 & 214312 \\
\hline Goods bought for resale without processing & ZABZ & 155608 & 161304 & 165667 & 169676 & 175971 \\
\hline Energy and water products for own consumption & ZACA & 1918 & 2048 & 2191 & 2522 & 3048 \\
\hline Goods and materials & $\mathrm{ZACB}$ & 3845 & 3917 & 4166 & 4271 & 4587 \\
\hline Hiring, leasing or renting of plant, machinery & & & & & & \\
\hline and vehicles & ZACC & 924 & 946 & 727 & 576 & 602 \\
\hline Commercial insurance premiums & ZACD & 827 & 1001 & 1061 & 1034 & 937 \\
\hline Road transport services & ZACE & 2137 & 2545 & 2557 & 2737 & 2499 \\
\hline Telecommunication services & ZACF & 561 & 624 & 626 & 607 & 571 \\
\hline Computer and related services & ZACG & 756 & 765 & 915 & 816 & 829 \\
\hline Advertising and marketing services & $\mathrm{ZACH}$ & 3047 & 3378 & 3298 & 3540 & 3601 \\
\hline Other services & ZACI & 16252 & 17642 & 18565 & 19620 & 21667 \\
\hline Taxes, duties and levies & ZACJ & 4576 & 4715 & 4896 & 5990 & 6361 \\
\hline National non-domestic (business) rates & ZACK & 3726 & 3859 & 3937 & 4336 & 4703 \\
\hline Cost of acquisitions & $\mathrm{ZACM}$ & 9355 & 8776 & 9936 & 10138 & 10065 \\
\hline Proceeds from disposals & ZACN & 1240 & 1328 & 1590 & 1516 & 1761 \\
\hline Net capital expenditure & $\mathrm{ZACO}$ & 8115 & 7448 & 8346 & 8622 & 8304 \\
\hline Amount included in acquisitions for assets under & & & & & & \\
\hline finance leasing arrangements & $\mathrm{ZACP}$ & 587 & 304 & 332 & 303 & 443 \\
\hline Work of a capital nature carried out by own staff & & & & & & \\
\hline (included in acquisitions) & ZACQ & 128 & 142 & 149 & 176 & 189 \\
\hline \multicolumn{7}{|l|}{ Gross margin } \\
\hline Amount & ZACR & 83708 & 88904 & 93830 & 95667 & 102417 \\
\hline As a percentage of adjusted turnover ${ }^{4}$ & ZACS & 34.9 & 35.6 & 36.2 & 36.0 & 36.7 \\
\hline Approximate gross value added at basic prices & ZACT & 53545 & 56104 & 59764 & 60020 & 64157 \\
\hline
\end{tabular}


2003

2004

2005

\section{Total turnover}

\section{Retail turnover}

1 Fruit (including fresh, chilled, dried, frozen, canned and processed)

2 Vegetables (including fresh, chilled, dried, frozen, canned and processed)

3 Meat (including fresh, chilled, smoked, frozen, canned and processed)

4 Fish, crustaceans and molluscs (including fresh, chilled, frozen, canned and processed)

5 Bakery products and cereals (including rice and pasta products)

6 Sugar, jam, honey, chocolate and confectionery (including ice-cream)

7 Alcoholic drink

8 Non-alcoholic beverages (including tea, coffee, fruit drinks and vegetable drinks)

9 Tobacco (excluding smokers requisites, eg pipes, lighters, etc)

10 Milk, cheese and eggs (including yoghurts and cream)

11 Oils and fats (including butter and margarine)

12 Food products not elsewhere classified (including sauces, herbs, spices and soups)

13 Pharmaceutical products

14 National Health Receipts

15 Other medical products and therapeutic appliances and equipment

16 Other appliances, articles and products for personal care

17 Other articles of clothing, accessories for making clothing

18 Garments

19 Footwear (excluding sports shoes)

20 Travel goods and other personal effects not elsewhere classified

21 Household textiles (including furnishing fabrics, curtains, etc)

22 Household and personal appliances whether electric or not

23 Glassware, tableware and household utensils (including non-electric)

24 Furniture and furnishings

25 Audio and visual equipment (including radios, televisions and video recorders)

26 Recording material for pictures and sound (including audio and video tapes, blank and pre-recorded records, etc)

27 Information processing equipment (including printers, software, calculators and typewriters)

28 Decorating and DIY supplies

29 Tools and equipment for house and garden

30 Books

31 Newspapers and periodicals

32 Stationery and drawing materials and miscellaneous printed matter

33 Carpets and other floor coverings (excluding bathroom mats, rush and door mats)

34 Photographic and cinematographic equipment and optical instruments

35 Telephone and telefax equipment (including mobile phones)

36 Jewellery, silverware and plate; watches and clocks

37 Works of art and antiques (including furniture, floor coverings and jewellery)

38 Equipment and accessories for sport, camping, recreation and musical instruments

39 Spare part and accessories for all types of vehicle and sales of bicycles

40 Games, toys, hobbies (including video game software, video game computers that plug into the tv, video-games cassettes and CD-ROMs)

41 Other goods not elsewhere classified (including sale of new postage stamps and sales of liquid and solid fuels)

42 Non-durable household goods (including household cleaning, maintenance products) and paper products and other non-durable household goods

43 Natural or artificial plants and flowers

44 Pets and related products (including pet food)

45 Repair of household and personal items

1 See chapter text.

2 Inclusive of VAT.

3 Exclusive of VAT.

4 Turnover is adjusted to take out VAT.

\begin{tabular}{|c|c|c|c|c|c|}
\hline ZABL & 265577 & 278373 & 288716 & 295952 & 310150 \\
\hline ZABN & 238456 & 250849 & 258903 & 264427 & 277298 \\
\hline DSSX & З 997 & 4507 & 4499 & 4657 & 5279 \\
\hline DSSY & 6871 & 8354 & 8470 & 9205 & 9077 \\
\hline DSSZ & 11671 & 13505 & 13727 & 13520 & 14876 \\
\hline DSTA & 2299 & 2549 & 2671 & 2866 & 2858 \\
\hline DSTC & 9661 & 12314 & 11880 & 12967 & 14151 \\
\hline DSTD & 6469 & 6446 & 6534 & 6406 & 7528 \\
\hline DSTE & 11301 & 12297 & 12931 & 13099 & 14035 \\
\hline DSTF & 6476 & 6713 & 7386 & 7514 & 7379 \\
\hline DSTG & 9016 & 9204 & 9020 & 8960 & 9139 \\
\hline DSTH & 7233 & 7390 & 7995 & 8356 & 8957 \\
\hline DSTI & 1222 & 1162 & 1287 & 1329 & 1295 \\
\hline DSTJ & 9185 & 4112 & 4399 & 4327 & 4572 \\
\hline DSTK & 2911 & 2963 & 2987 & 3090 & 3 370 \\
\hline DSTL & 7740 & 8647 & 9006 & 9673 & 9966 \\
\hline DSTN & 2753 & 3122 & 3388 & 3387 & 3758 \\
\hline DSTO & 9611 & 10698 & 11111 & 11405 & 12314 \\
\hline DSTP & 1293 & 1955 & 2089 & 2452 & 2834 \\
\hline DSTQ & 28331 & 29691 & 30375 & 31294 & 31654 \\
\hline DSTR & 5270 & 5622 & 5904 & 6037 & 6559 \\
\hline DSTT & 1007 & 1175 & 1120 & 1124 & 1332 \\
\hline DSTV & 3656 & 3799 & 3890 & 3700 & 3848 \\
\hline DSUA & 6580 & 6776 & 6798 & 6957 & 6956 \\
\hline DSUB & 2823 & 2843 & 2743 & 3124 & 3024 \\
\hline DSUC & 12094 & 13285 & 13493 & 13620 & 13998 \\
\hline DSUE & 4781 & 4818 & 5130 & 5646 & 6211 \\
\hline DSUG & 3591 & 3788 & 4488 & 4366 & 4000 \\
\hline DSUL & 3056 & 3077 & 3743 & 3600 & 3704 \\
\hline DSUM & 6548 & 6631 & 7427 & 6928 & 5652 \\
\hline DSUN & 3007 & 3 452 & 2838 & 2983 & 3187 \\
\hline DSUP & 2752 & 2748 & 3004 & 2646 & 3017 \\
\hline DSUQ & 3709 & 4067 & 4053 & 4048 & 4244 \\
\hline DSUW & 3864 & 3824 & 3989 & 4201 & 4583 \\
\hline DSUX & 3411 & 3757 & 3386 & 3526 & 3458 \\
\hline DSUZ & 1402 & 1670 & 1842 & 2052 & 2078 \\
\hline DSVA & 2238 & 2293 & 3272 & 3476 & 3480 \\
\hline DSVB & 4387 & 4312 & 4681 & 4697 & 5347 \\
\hline DSVF & 1509 & 1493 & 1619 & 1720 & 1568 \\
\hline DSVH & 3624 & 3803 & 3732 & 3612 & 4431 \\
\hline DSVI & 1082 & 572 & 610 & 556 & 768 \\
\hline DSVM & 5468 & 5962 & 5920 & 5745 & 5933 \\
\hline DSVN & 3359 & 3134 & 2884 & 2623 & 3861 \\
\hline DSVO & 4405 & 5017 & 5429 & 5291 & 4809 \\
\hline DSVQ & 3266 & 3745 & 3117 & 3253 & 3810 \\
\hline DSVR & 2497 & 2681 & 3012 & 3228 & 3314 \\
\hline DSVS & 1031 & 875 & 1021 & 1161 & 1081 \\
\hline
\end{tabular}

Source: Office for National Statistics: 01633456592 
Service industry

\section{$24.2 \begin{aligned} & \text { Retail trade: index numbers of value and volume of sales } \\ & \text { Great Britain }\end{aligned}$ \\ Not seasonally adjusted}

Weekly average $(2000=100)$

\begin{tabular}{|c|c|c|c|c|c|c|c|c|c|c|c|c|c|}
\hline & & & 1998 & 1999 & 2000 & 2001 & 2002 & 2003 & 2004 & 2005 & 2006 & 2007 & 2008 \\
\hline Value & & $\begin{array}{l}\text { Sales in } \\
2000 \\
£ \text { million }\end{array}$ & & & & & & & & & & & \\
\hline $\begin{array}{l}\text { All retailing } \\
\text { Large } \\
\text { Small }\end{array}$ & $\begin{array}{l}\text { EAFY } \\
\text { EAFZ } \\
\text { EAGA }\end{array}$ & $\begin{array}{c}207149 \\
153022 \\
54128\end{array}$ & $\begin{array}{l}93.4 \\
91.7 \\
98.6\end{array}$ & $\begin{array}{l}96.5 \\
95.8 \\
98.5\end{array}$ & $\begin{array}{l}100.0 \\
100.0 \\
100.0\end{array}$ & $\begin{array}{l}105.9 \\
106.5 \\
104.1\end{array}$ & $\begin{array}{l}110.6 \\
112.2 \\
105.8\end{array}$ & $\begin{array}{l}113.7 \\
118.2 \\
101.1\end{array}$ & $\begin{array}{l}118.8 \\
123.9 \\
104.1\end{array}$ & $\begin{array}{l}119.9 \\
126.0 \\
102.8\end{array}$ & $\begin{array}{l}123.3 \\
130.7 \\
102.2\end{array}$ & $\begin{array}{l}127.8 \\
136.1 \\
104.5\end{array}$ & $\begin{array}{l}131.7 \\
140.0 \\
108.4\end{array}$ \\
\hline $\begin{array}{l}\text { non-food stores } \\
\text { Textiles, clothing, footwear and }\end{array}$ & EAGE & 18781 & 92.6 & 95.2 & 100.0 & 105.0 & 107.4 & 109.2 & 111.2 & 110.8 & 114.0 & 117.9 & 116.0 \\
\hline $\begin{array}{l}\text { leather } \\
\text { Household goods stores } \\
\text { Other specialised non-food stores }\end{array}$ & $\begin{array}{l}\text { EAFU } \\
\text { EAFV } \\
\text { EAFW }\end{array}$ & $\begin{array}{l}27880 \\
27699 \\
31999\end{array}$ & $\begin{array}{l}93.8 \\
91.6 \\
93.1\end{array}$ & $\begin{array}{l}96.0 \\
95.7 \\
96.8\end{array}$ & $\begin{array}{l}100.0 \\
100.0 \\
100.0\end{array}$ & $\begin{array}{l}108.4 \\
107.6 \\
105.7\end{array}$ & $\begin{array}{l}114.9 \\
113.1 \\
110.5\end{array}$ & $\begin{array}{l}118.9 \\
113.4 \\
115.6\end{array}$ & $\begin{array}{l}124.7 \\
117.2 \\
123.0\end{array}$ & $\begin{array}{l}126.5 \\
112.9 \\
123.0\end{array}$ & $\begin{array}{l}131.8 \\
114.2 \\
123.5\end{array}$ & $\begin{array}{l}136.4 \\
118.0 \\
128.0\end{array}$ & $\begin{array}{l}136.4 \\
114.7 \\
134.2\end{array}$ \\
\hline $\begin{array}{l}\text { Other retail sale (non-store) and } \\
\text { repair }\end{array}$ & EAFX & 11749 & 97.1 & 98.8 & 100.0 & 97.0 & 100.5 & 96.2 & 102.7 & 99.1 & 100.6 & 108.6 & 116.2 \\
\hline \multicolumn{14}{|l|}{ Volume } \\
\hline All retailing & EAHC & 207149 & 92.5 & 95.7 & 100.0 & 106.1 & 112.2 & 116.3 & 123.3 & 125.8 & 129.8 & 135.3 & 139.7 \\
\hline Predominantly food stores & EAGW & 89041 & 95.5 & 97.2 & 100.0 & 104.1 & 108.2 & 111.9 & 116.5 & 119.7 & 122.7 & 124.2 & 125.7 \\
\hline $\begin{array}{l}\text { Predominantly non-food stores } \\
\text { Non specialised predominantly }\end{array}$ & EAGX & 106359 & 89.9 & 94.3 & 100.0 & 108.5 & 116.2 & 121.3 & 129.6 & 131.8 & 136.4 & 144.0 & 149.1 \\
\hline $\begin{array}{l}\text { non-food stores } \\
\text { Textiles,clothing, footwear and }\end{array}$ & EAHI & 18781 & 91.5 & 94.0 & 100.0 & 106.0 & 110.5 & 113.8 & 118.0 & 119.3 & 124.1 & 129.7 & 130.3 \\
\hline leather & EAGY & 27880 & 88.8 & 92.9 & 100.0 & 112.1 & 123.7 & 129.6 & 139.3 & 143.9 & 150.9 & 156.9 & 161.6 \\
\hline Household goods stores & EAGZ & 27699 & 85.8 & 92.6 & 100.0 & 109.6 & 117.8 & 122.3 & 130.8 & 131.2 & 137.7 & 148.5 & 151.3 \\
\hline Other specialised non-food stores & EAHA & 31999 & 93.6 & 97.1 & 100.0 & 105.9 & 111.6 & 117.5 & 127.0 & 129.1 & 130.0 & 137.2 & 147.3 \\
\hline $\begin{array}{l}\text { Other retail sale (non-store) } \\
\text { and repair }\end{array}$ & EAHB & 11749 & 93.2 & 96.2 & 100.0 & 99.6 & 106.5 & 105.4 & 117.1 & 118.0 & 123.6 & 141.1 & 161.4 \\
\hline
\end{tabular}




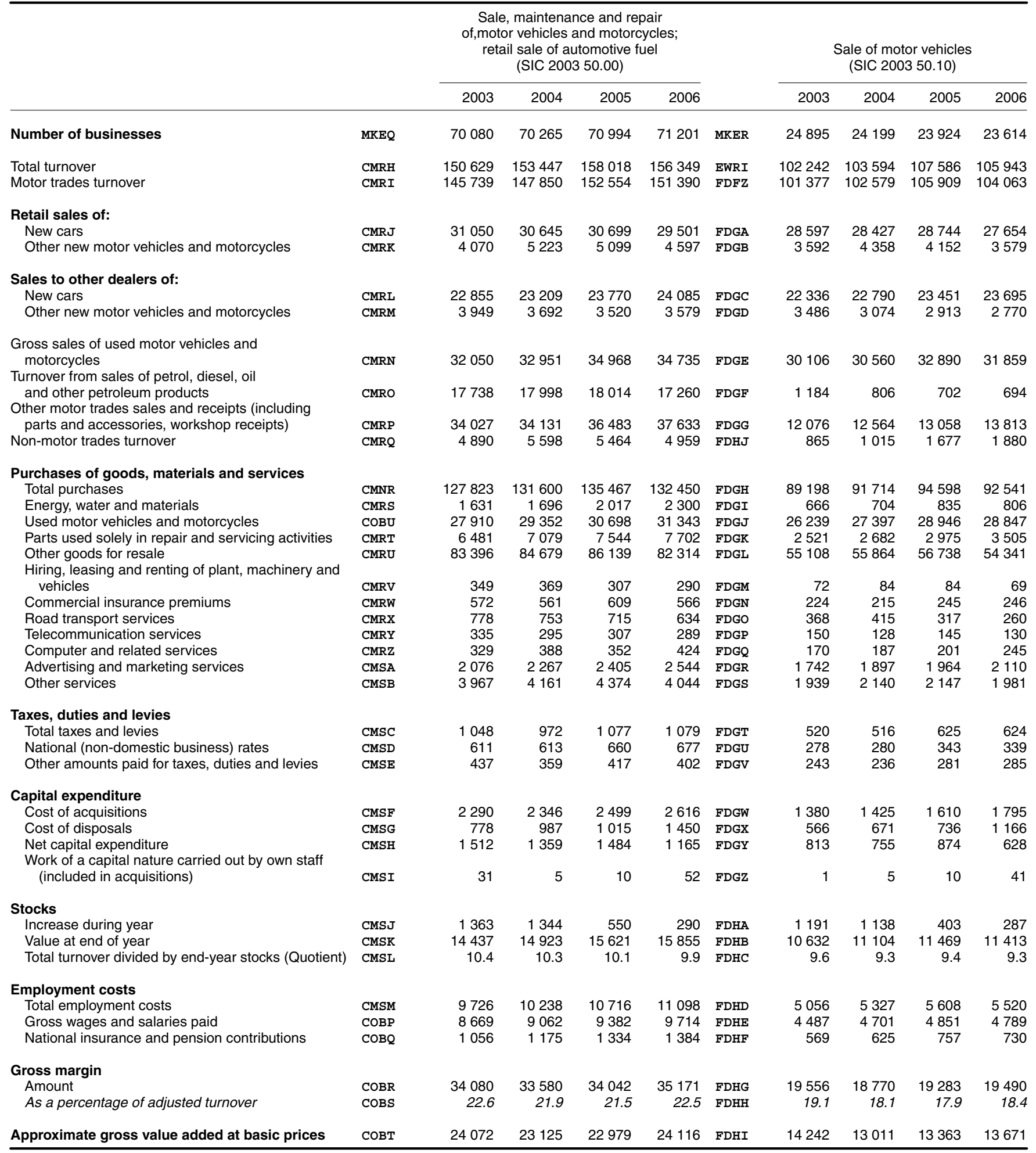




\begin{tabular}{|c|c|c|c|c|c|c|c|c|c|c|}
\hline & & & $\begin{array}{l}\text { aintenanc } \\
\text { of motor } \\
\text { (SIC 20) }\end{array}$ & $\begin{array}{l}\text { and rep } \\
\text { vehicles } \\
350.20 \text { ) }\end{array}$ & & & & $\begin{array}{l}\text { of moto } \\
\text { and acc } \\
\text { (SIC 20 }\end{array}$ & $\begin{array}{l}\text { vehicle p } \\
\text { ssories } \\
350.30 \text { ) }\end{array}$ & \\
\hline & & 2003 & 2004 & 2005 & 2006 & & 2003 & 2004 & 2005 & 2006 \\
\hline Number of businesses & MKES & 29188 & 30050 & 30973 & 31666 & MKET & 7799 & 7952 & 8067 & 8096 \\
\hline Total turnover & FDHK & 12542 & 13337 & 14445 & 14261 & FDIW & 13801 & 13694 & 13659 & 14428 \\
\hline Motor trades turnover & FDHL & 12147 & 13023 & 14082 & 13992 & FDIX & 13304 & 12702 & 12722 & 13426 \\
\hline Retail sales of: & & & & & & & & & & \\
\hline New cars & FDHM & 1081 & 939 & 1000 & 768 & FDIY & 1168 & 1241 & 880 & 904 \\
\hline Other new motor vehicles and motorcycles & FDHN & 153 & 213 & 164 & 283 & FDIZ & .. & 172 & 233 & 206 \\
\hline Sales to other dealers of: & & & & & & & & & & \\
\hline New cars & FDHO & 8 & .. & 23 & 40 & FDJA & 509 & .. & 294 & 322 \\
\hline Other new motor vehicles and motorcycles & FDHP & 3 & .. & .. & 48 & FDJB & .. & .. & 75 & 51 \\
\hline Turnover from sales of petrol, diesel, oil & & & & & & & & & & \\
\hline and other petroleum products & FDHR & 117 & 100 & 119 & 240 & FDJD & 70 & 86 & 1 & 4 \\
\hline Other motor trades sales and receipts (including & & & & & & & & & & \\
\hline parts and accessories, workshop receipts) & FDHS & 9674 & 10386 & 11500 & 11298 & FDJE & 11085 & 10362 & 10987 & 11447 \\
\hline Non-motor trades turnover & FDHT & 396 & 314 & 363 & 269 & FDJF & 497 & 992 & 936 & 1002 \\
\hline Purchases of goods, materials and services & & & & & & & & & & \\
\hline Total purchases & FDHU & 8100 & 8795 & 9406 & 9045 & FDJG & 11062 & 10958 & 11223 & 11736 \\
\hline Energy, water and materials & FDHV & 572 & 652 & 735 & 897 & FDJH & 220 & 227 & 329 & 439 \\
\hline Used motor vehicles and motorcycles & FDHW & 897 & 1041 & 1046 & 1134 & FDJI & .. & 371 & 272 & 387 \\
\hline Parts used solely in repair and servicing activities & FDHX & 3374 & 3980 & 4184 & 3909 & FDJU & 410 & 183 & 208 & 158 \\
\hline Other goods for resale & FDHY & 1774 & 1453 & 1739 & 1591 & FDJK & .. & 9000 & 8976 & 9268 \\
\hline Hiring, leasing and renting of plant, machinery and & & & & & & & & & & \\
\hline vehicles & FDHZ & 77 & 111 & 120 & 140 & FDJL & 151 & 136 & 62 & 53 \\
\hline Commercial insurance premiums & FDIA & 204 & 227 & 242 & 198 & FDJM & 89 & 78 & 84 & 82 \\
\hline Road transport services & FDIB & 49 & 104 & 79 & 39 & FDJN & 267 & 126 & 207 & 237 \\
\hline Telecommunication services & FDIC & 97 & 103 & 97 & 88 & FDJO & 60 & 45 & 45 & 44 \\
\hline Computer and related services & FDID & 61 & 72 & 39 & 55 & FDJP & 75 & 107 & 96 & 104 \\
\hline Advertising and marketing services & FDIE & 127 & 125 & 111 & 86 & FDJQ & 165 & 208 & 290 & 274 \\
\hline Taxes, duties and levies & & & & & & & & & & \\
\hline Total taxes and levies & FDIG & 197 & 215 & 218 & 219 & FDJS & 102 & 116 & 128 & 135 \\
\hline National (non-domestic business) rates & FDIH & 162 & 152 & 146 & 167 & FDJT & 84 & 89 & 96 & 106 \\
\hline Other amounts paid for taxes, duties and levies & FDII & 35 & 63 & 72 & 52 & FDJU & 19 & 27 & 32 & 29 \\
\hline Capital expenditure & & & & & & & & & & \\
\hline Cost of acquisitions & FDIJ & 433 & 442 & 441 & 348 & FDJV & 221 & 199 & 194 & 210 \\
\hline Cost of disposals & FDIK & 87 & 98 & 109 & 93 & FDJW & 47 & 54 & 76 & 97 \\
\hline Net capital expenditure & FDIL & 346 & 345 & 332 & 255 & FDJX & 173 & 144 & 118 & 113 \\
\hline $\begin{array}{l}\text { Work of a capital nature carried out by own staff } \\
\text { (included in acquisitions) }\end{array}$ & Tח & & & & & FDTY & 29 & & & 6 \\
\hline & & & & & & & & & & \\
\hline Stocks & & & & & & & & & & \\
\hline Increase during year & FDIN & 102 & 36 & -13 & 7 & FDJZ & 76 & 81 & 134 & 70 \\
\hline Value at end of year & FDIO & 881 & 835 & 958 & 1085 & FDKA & 1750 & 1807 & 1818 & 2047 \\
\hline Total turnover divided by end-year stocks (Quotient) & FDIP & 14.2 & 16.0 & 15.1 & 13.1 & FDKB & 7.9 & 7.6 & 7.5 & 7.0 \\
\hline Employment costs & & & & & & & & & & \\
\hline Total employment costs & FDIQ & 2480 & 2581 & 2697 & 2927 & FDKC & 1427 & 1565 & 1618 & 1773 \\
\hline Gross wages and salaries paid & FDIR & 2233 & 2315 & 2429 & 2635 & FDKD & 1268 & 1391 & 1423 & 1556 \\
\hline National insurance and pension contributions & FDIS & 247 & 266 & 268 & 292 & FDKE & 159 & 174 & 195 & 217 \\
\hline Gross margin & & & & & & & & & & \\
\hline Amount & FDIT & 6597 & 6857 & 7415 & 7609 & FDKF & 4501 & 4199 & 4313 & 4671 \\
\hline As a percentage of adjusted turnover & FDIU & 52.6 & 51.4 & 51.3 & 53.4 & FDKG & 32.6 & 30.7 & 31.6 & 32.4 \\
\hline Approximate gross value added at basic prices & FDIV & 4552 & 4548 & 4980 & 5200 & FDKH & 2831 & 2796 & 2547 & 2753 \\
\hline
\end{tabular}




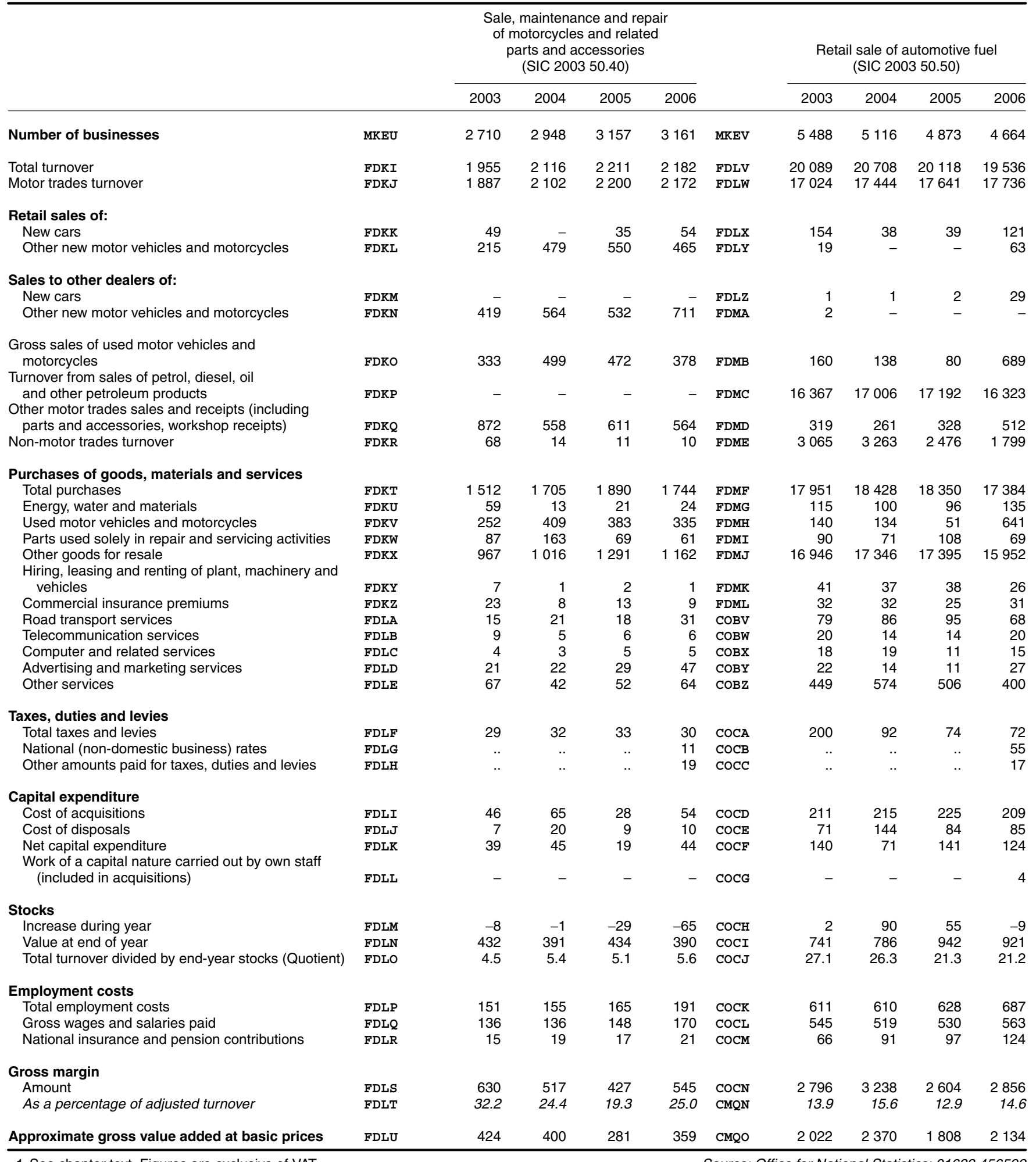

1 See chapter text. Figures are exclusive of VAT. 
Service industry

$£$ million and percentages

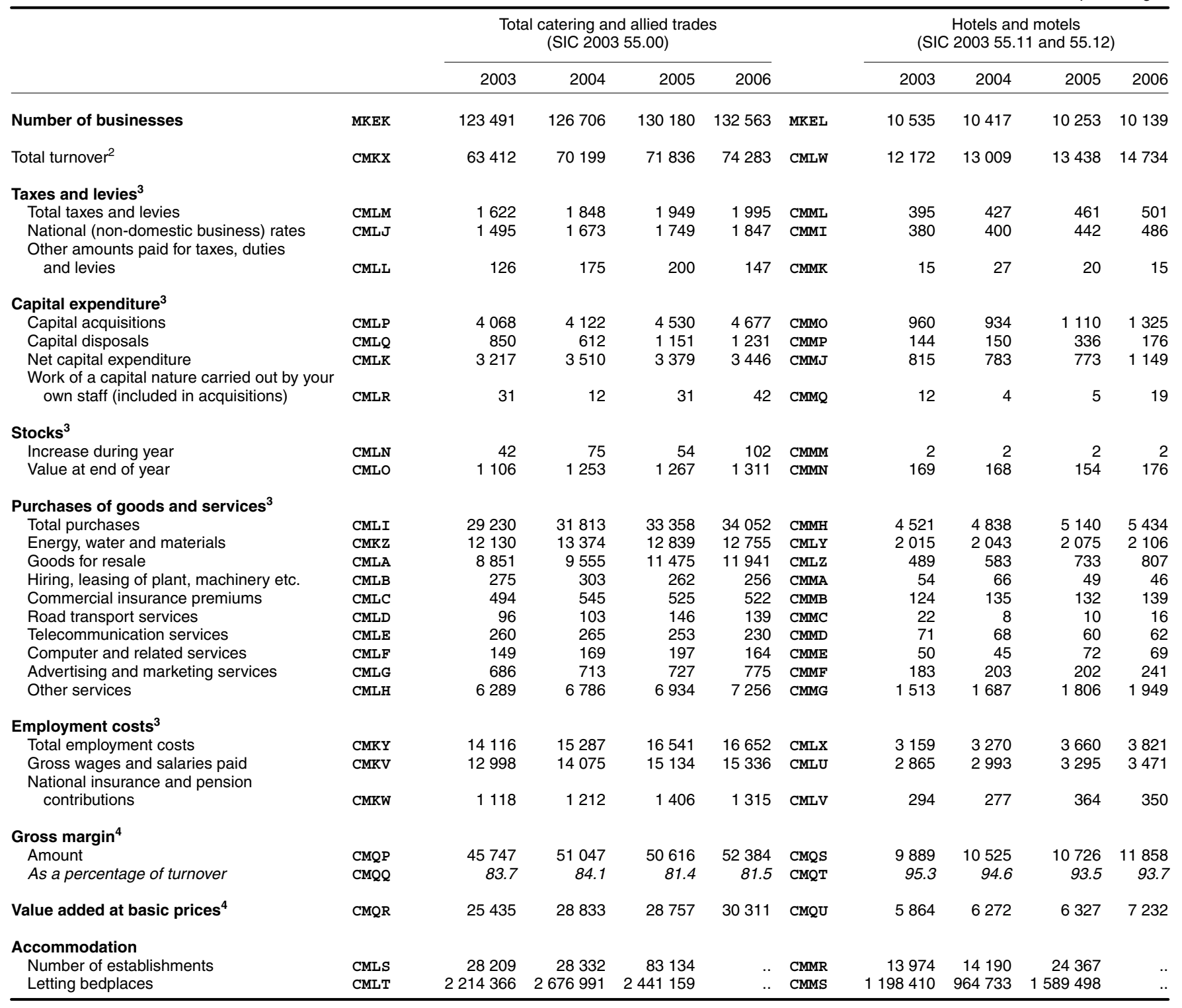




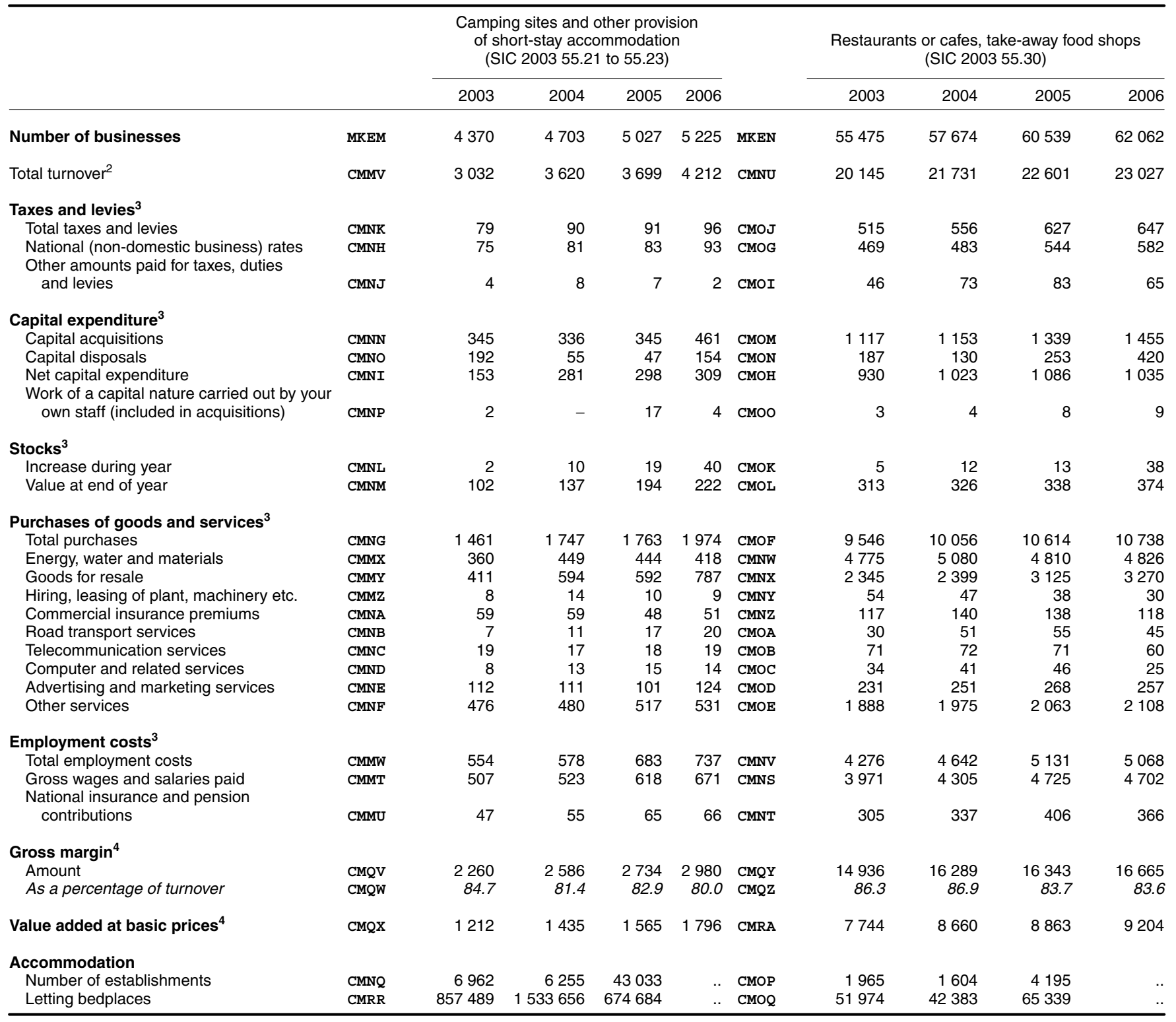


Service industry

$£$ million and percentages

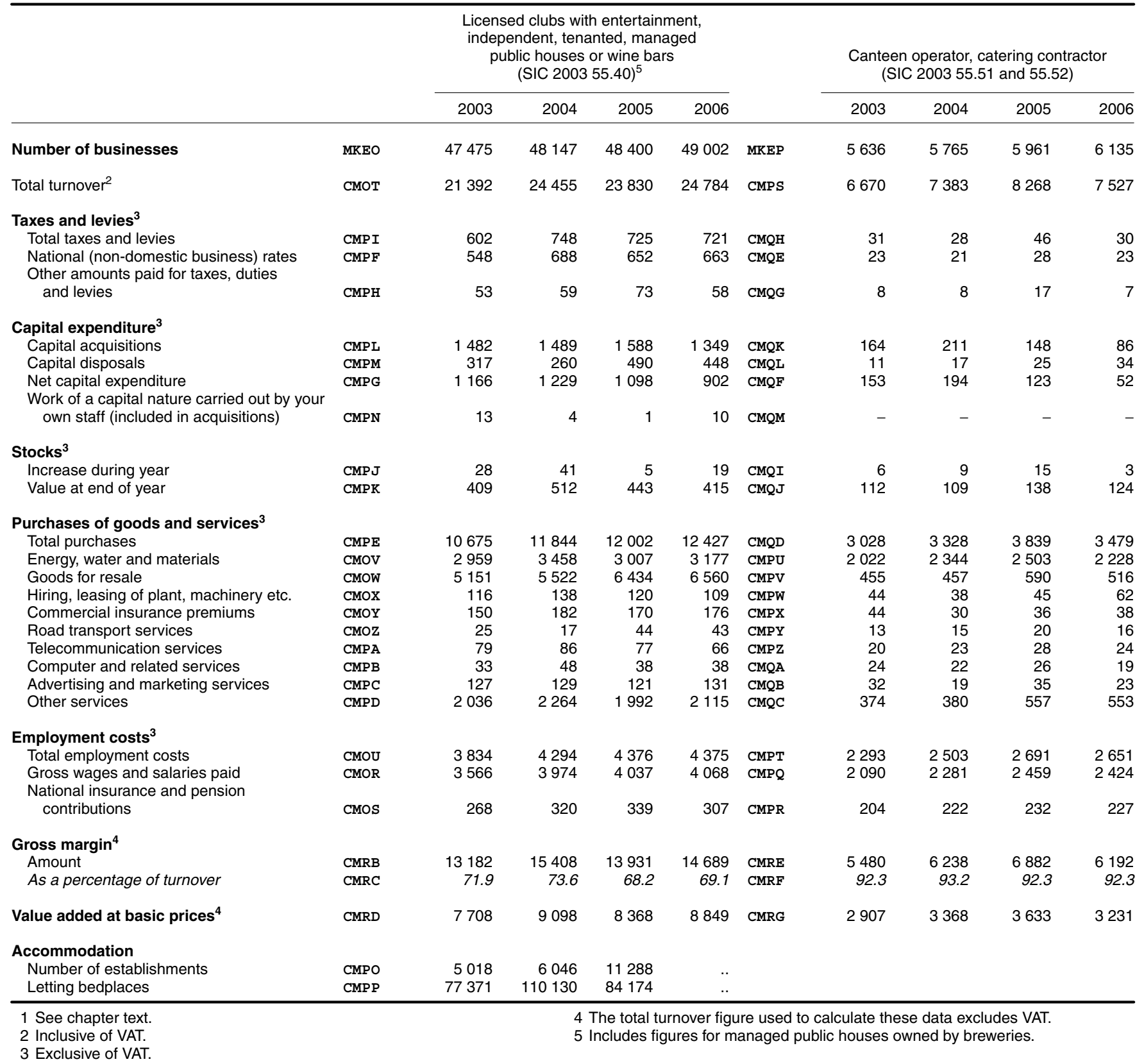

Source: Office for National Statistics: 01633456592 